\title{
Assessing Disease Activity in Psoriatic Arthritis: A Literature Review
}

\author{
Laura J. Tucker · Laura C. Coates · Philip S. Helliwell
}

Received: October 5, 2018 / Published online: November 23, 2018

(C) The Author(s) 2018

\begin{abstract}
Psoriatic arthritis (PsA) is a multifaceted disease, with a high impact on patients' psychological and physical well-being. There is increasing recognition that assessment of both clinical aspects of disease and patient identified concerns, such as fatigue, work disability, and treatment satisfaction need to be addressed. Only then can we fully understand disease burden and make well-informed treatment decisions aimed at improving patients' lives. In recent years, there has been much progress in the development of unidimensional and composite measures of disease activity, as well as questionnaires capturing the patient's perspective in psoriatic disease. Despite these advances, there remains disagreement amongst clinicians as to which instruments should be used. As a consequence, they are yet to receive widespread implementation in routine clinical practice.
\end{abstract}

Enhanced digital features To view enhanced digital features for this article go to https://doi.org/10.6084/ m9.figshare.7338887.

L. J. Tucker · L. C. Coates ( $\square)$

Nuffield Department of Orthopaedics,

Rheumatology and Musculoskeletal Sciences,

University of Oxford, Oxford, UK

e-mail: laura.coates@ndorms.ox.ac.uk

P. S. Helliwell

Leeds Institute of Rheumatic and Musculoskeletal

Medicine, University of Leeds, Leeds, UK
This review aims to summarize currently available clinical and patient-derived assessment tools, which will provide clinicians with a practical and informative resource.

Keywords: Composite measures; Disease activity; Impact of disease; Outcome measures; Patient perspective; Psoriatic arthritis; Treatment satisfaction; Work disability

\section{INTRODUCTION}

Psoriatic arthritis (PsA) is a heterogeneous disease with variable involvement of a number of disease "domains". These include peripheral arthritis, psoriasis (skin and nail), enthesitis, dactylitis, and axial disease. In addition to considering the physician-assessed domains of disease activity, the overall impact of the disease on individual patients may encompass additional issues such as pain, loss of function, and impairment of quality of life. When designing clinical studies in PsA, all of these different aspects of the disease may be important. To formalize this concept, the Group for Research and Assessment of Psoriasis and Psoriatic Arthritis/Outcome Measures in Rheumatology (GRAPPA-OMERACT) has developed a core domain set for PsA which highlights the domains relevant to this disease. The "inner circle" of the core set is recommended to be 
measured in all clinical trials and includes musculoskeletal and skin disease activity, pain, patient global assessment, physical function, health related quality of life (HRQoL), fatigue, and systemic inflammation. Additional domains considered important were economic cost, emotional well-being, participation, and structural damage [1].

The TIght Control Of Psoriatic Arthritis (TICOPA) trial confirmed the benefit of regular disease activity assessment using objective outcome measures [2]. As a result of this, new recommendations for the management of PsA focus on the importance of regular assessment of disease activity, as well as disease impact from the patient's perspective to guide therapeutic decisions [3-5]. Thus, the use of objective outcome measures is now becoming more routine in clinical practice outside of the research setting.

In this review, we summarize data for measures of individual disease domains, as well as newer composite measures of disease activity in general, with a focus on measures that may be practical and informative in routine clinical use.

This article is based on previously conducted studies and does not contain any studies with human participants or animals performed by any of the authors.

\section{INDIVIDUAL DOMAIN MEASURES}

\section{Arthritis}

All measures of peripheral arthritis are based on tender and swollen joint counts but are often combined with some patient-reported outcomes to give a composite measure. Given the heterogeneity of joint involvement in PsA, a more complete $68 / 66$ joint counts is required rather than reduced joint counts in rheumatoid arthritis (RA), which can miss significant amounts of active disease [6]. Many studies in PsA have used the disease activity score (DAS) borrowed from RA to assess arthritis, but the reduced joint count within the DAS28 in particular is a concern and the cut points used in RA have not been validated in PsA.
In clinical trials, the primary outcome of new drug randomized controlled trials (RCTs) has always been the American College of Rheumatology 20\% (ACR20) response. The ACR response criteria are borrowed from RA but are usually modified to use a $68 / 66$ joint count. They are reported as a binary outcome for those who achieve a 20,50 , or $70 \%$ improvement in tender and swollen joint counts, plus three of the following: physician global, patient global, patient pain, function, and $C$ reactive protein (CRP)/erythrocyte sedimentation rate (ESR). While these perform well at differentiating drug and placebo in polyarticular patients [7], they may not perform as well in oligoarticular patients and are not particularly feasible in the clinic.

More recently the disease activity in PsA (DAPSA) has been developed from the disease activity in reactive arthritis (DAREA) scale [8]. This includes a 68/66 joint count summed with a patient global, patient pain score, and CRP level. The DAPSA provides a continuous score of arthritis activity and has validated cut points for remission $(<4)$ and low disease activity $(<14)$ [9]. DAPSA disease activity states have been shown to correlate with functional status and structural progression on radiographs providing further evidence of their validity [10]. However, research to date has focused on polyarticular patients where disease activity is proportional to joint counts but may be less representative in oligoarthritis patients where the DAPSA high disease activity level is unlikely to ever be reached despite high impact of disease.

\section{Enthesitis}

There are two key clinical measures of enthesitis in PsA that have been shown to be valid. During development, the sites for the Leeds Enthesitis Index (LEI) were chosen by reducing the Mander entheseal sites to those most commonly tender in PsA patients. It includes just three sites bilaterally (lateral epicondyles, medial condyles of the femur, and Achilles tendons) [11]. The second measure, the Spondyloarthritis Consortium of Canada (SPARCC) enthesitis score is composed of sites that were found to be 
commonly involved in the imaging of spondyloarthritis (PsA and ankylosing spondylitis (AS)) patients. It contains 16 sites (eight bilaterally) with some overlap with the LEI [12]. Both scores have been used in a number of PsA clinical trials and as of yet there is no clear superiority of either measure. However, it should be noted that clinical assessment of pain at entheseal insertions has been shown to have a poor correlation with ultrasound evidence of enthesitis [13]. For this reason, current studies investigating treatment of enthesitis as a primary or key secondary outcome have also included imaging outcome measures to provide additional face validity.

\section{Dactylitis}

Dactylitis is a typical feature of PsA where inflammation occurs in multiple tissues within a single digit. In the majority of clinical studies, dactylitis has been assessed by a simple count of dactylitic digits, sometimes multiplied by the 0-3 tenderness grading used in the Ritchie index. While these simple counts can show sensitivity in clinical trials, a more detailed quantified scoring system called the Leeds Dactylitis Instrument (LDI) has also been developed [14]. The dactylometer measures the circumference of swollen digits at the base of the finger. This provides a quantifiable definition of dactylitis-an increase in circumference of $>10 \%$ more than the contralateral digit. The LDI as a measure then combines this level of swelling using the circumference measurement with a score (either $0-3$ or $0 / 1$ ) for tenderness [14]. The LDI score has been shown to be responsive in clinical trials and has a larger effect size than simple dactylitis counts $[14,15]$.

\section{Axial Disease}

Axial disease in PsA is probably the least well defined aspect of the disease. There are also significant issues with the outcome measures used in axial PsA. The outcomes tested have all been adopted from AS. The Bath AS Disease Activity Index (BASDAI) has been used to measure disease activity in axial PsA and has been shown to correlate with other disease activity measures. However, it is not able to differentiate between axial and peripheral musculoskeletal disease activity with high scores in those with active peripheral PsA only. This lack of specificity limits its use, as it will not be able to differentiate individual domains of disease activity.

In recent years, the AS Disease Activity Score (ASDAS) in axial SpA has been developed. This composite score includes a number of questions from the BASDAI combined with the CRP result. This score has been validated in axial SpA including in patients with axial PsA $[16,17]$ but due to its components, it is likely to be affected by the same problem as the BASDAI, with the scores being affected by peripheral disease activity.

\section{Skin}

The most commonly used outcome measure in psoriasis clinical trials is the Psoriasis Area and Severity Index (PASI), which is a composite of body surface area (BSA), erythema, induration, and scaling of psoriasis on different areas of the body (head, trunk, upper limbs, lower limbs) [18]. Although it is often used in some healthcare settings, it is relatively burdensome in clinical practice. Additionally, in PsA patients who have mild skin disease, it may be representative of disease burden and less responsive to change.

Simpler measures have focused on only measuring body surface area or have used simpler assessments of overall disease activity, such as the static Physician Global Assessment (sPGA). This can be recorded on a scale of 1 (clear) to 6 (very severe) but assesses plaque quality without assessing body surface area. More recently, work has been undertaken looking at a combination of the two-sPGA $\times$ BSA to allow a simple feasible measure that encompasses both body surface area and a characterization of the psoriasis plaque [19]. Validation work has shown that this simpler measure shows good concordance with PASI and could be used as a PASI proxy in routine clinical practice [20]. 


\section{COMPOSITE MEASURES OF DISEASE ACTIVITY}

Given that PsA is so heterogeneous with involvement of multiple domains in each patient, a number of composite measures of psoriatic disease have been developed to measure the overall disease burden.

The first of these is a measure of disease state developed specifically to be a target of therapy. The minimal disease activity (MDA) criteria encompass seven different items that are assessed individually. The criteria state that patients are in MDA if they achieve five of the seven criteria: tender joint count $\leq 1$, swollen joint count $\leq 1$, enthesitis count $\leq 1$, PASI $\leq 1$ or $\mathrm{BSA} \leq 3$, patient global visual analogue score $(\mathrm{VAS}) \leq 20 \mathrm{~mm}$, patient pain $\mathrm{VAS} \leq 15 \mathrm{~mm}$ and health assessment questionnaire (HAQ) $\leq 0.5$ [21]. The advantage of these criteria is that they assess multiple domains of disease in order to establish disease control; however, assess these individually rather than combining them into one single score. The MDA criteria have been shown to be associated with lower radiographic progression [22] and patient impact of disease [23], as well as improved quality of life, functional ability, and work stability [24]. They have been recommended as a target of therapy in PsA by the international treat-to-target (T2T) taskforce [5] and the GRAPPA-OMERACT group [25].

The principle limitation of the MDA criteria is that they only define a disease state and do not allow assessment of different levels of disease activity. The first true disease activity composite measure in PsA was the Composite Psoriatic Disease Activity Index (CPDAI) [26], which was based on the Group for Research and Assessment of Psoriasis and Psoriatic Arthritis (GRAPPA) treatment grid system [27]. Each domain of PsA (peripheral joints, skin, entheses, dactylitis, and axial disease) is assessed using a measure of disease activity and a measure of disease impact, and these are summed together to create a total score of $0-15$. The CPDAI correlates well with patient and physician global assessments [26] and was able to differentiate between different etanercept doses in the
Psoriasis Randomized Etanercept STudy in Subjects with Psoriatic Arthritis (PRESTA) trial [28], showing that it identifies other aspects of disease beyond the peripheral arthritis.

Two further measures were developed by the international GRAPPA group through a specific project called GRACE (GRAPPA Composite Exercise). This study used two different methodologies to analyze data on 503 patients assessed at baseline, 3, 6, and 12 months. The "gold standard" for disease activity was the decision of the physician to change or escalate treatment. The PsA Disease Activity Score (PASDAS) is a weighted index developed using similar methodology to the RA disease activity score. This includes seven components identified on principle component analysis (arthritis, enthesitis, dactylitis, CRP, and patient-reported outcomes). The GRACE index contains eight domain measures transformed using desirability functions and then combined [29]. Both of these new measures performed well in the development dataset [29] and in retrospective analyses of RCTs [30], including correlation with function and radiographic progression [31]. The effect sizes of these indices were greater than CPDAI, DAPSA, and DAS28, meaning that smaller sample sizes may be required for future trials using these measures. Candidate cut-offs for different levels of disease activity and response criteria have also been developed for the PASDAS, GRACE, and CPDAI [32].

\section{THE PATIENT PERSPECTIVE- PATIENT-REPORTED OUTCOME MEASURES (PROMS)}

In order to capture the full impact of psoriatic disease on an individual's physical and psychological well-being, there has been a paradigm shift in recent years towards the use of outcome measures which encompass real-life outcomes important to patients alongside the validated metrics already mentioned [25]. Patient-Reported Outcome Measures (PROMs) are derived directly from patients and have been found to be reliable indicators of disease status and predictors of long-term outcomes [33-35]. 
This review will now focus on tools available to assess the impact of psoriatic disease on patients, their ability to work, and satisfaction with treatment.

\section{Measuring the Impact of Psoriatic Disease from a Patient's Perspective}

The Psoriatic Arthritis Impact of Disease (PsAID) questionnaire was recently developed by the European League Against Rheumatism (EULAR) taskforce and aims to reflect the impact of PsA from the patient perspective [36]. This patientderived outcome measure is specific to PsA and is quick and simple to perform. It encompasses 12 domains, which address physical and psychological aspects of psoriatic disease, such as work, fatigue, and physical function. Each domain is assessed by a single question with patient response on a numeric rating scale $0-10$, with higher results indicating a more severe condition. Each domain carries a different weight, with pain having the greatest effect [36]. Two versions of the PsAID questionnaire have been developed: the 12-item questionnaire (PSAID12) for clinical practice and a nine-item questionnaire (PSAID9) for use in clinical trials. The cut-off value for a patient-acceptable symptom state is $\leq 4$ for both scores [36].

The PSAID questionnaire was validated in an international cross-sectional and longitudinal study of more than 470 patients, across 13 countries and was shown to have high reliability, generalizability, and strong correlation with patient global assessment (PGA) [36]. Holland et al. conducted a prospective cohort study of 129 patients with PsA to further investigate the validity of the PSAID questionnaire and determine the relationship between individual PsAID questions and established PROMs [37]. They concluded that both PSAID9 and PSAID12 questionnaires are reliable measures of impact of disease in PsA and are feasible in clinical practice. The PsAID questionnaire, and the individual items within it, strongly correlate with other PROMs [37]. While this lends support to the notion that conventional PROMs are in fact sensitive to patient concerns, the strong correlation of PsAID across a range of established PROMs could eliminate the need for multiple questionnaires, thereby reducing questionnaire burden for patients and improving feasibility in both clinical and research settings. Similar results were demonstrated in an Italian PsA cohort, with an emphasis on the importance of clinical judgement, in those patients with co-existent fibromyalgia, as this can influence the final PSAID score [38].

While the PsAID questionnaire is not a measure of disease activity, it does have the potential as an outcome measure to enable individualized treatment through the identification and targeting of those aspects of disease deemed most important to each patient. Although the PsAID questionnaire has been found to be sensitive to change $[36,37]$, independent cohorts and interventional clinical trials, including patients treated with biologic disease-modifying anti-rheumatic drugs (bDMARDs), are needed to further validate the PsAID questionnaire.

\section{Work Disability in Psoriatic Arthritis}

It is now recognized that work disability (WD) is an important functional outcome for PsA patients [36]. WD encompasses a spectrum of disabilities, ranging from absenteeism (time missed from work due to health reasons), presenteeism (reduced effectiveness at work due to health reasons), productivity loss (absenteeism plus presenteeism) through to unemployment. Levels of unemployment and WD are high in PsA, with unemployment levels ranging from 20 to $50 \%$ [39-45] and WD as high as 39\% [39], and translates into significant individual and societal economic costs [39, 41, 45, 46].

A large multicenter study of 400 PsA patients assessed WD using the Work Productivity and Activity Impairment (WPAI) questionnaire and found that of the 236 participants who were in work, absenteeism, presenteeism, and productivity loss rates were 14,39 , and $46 \%$, respectively. A further $26 \%$ of participants were unemployed. Greater age, recent onset of disease, and worse physical function were associated with higher risk of unemployment, while patient-reported employer helpfulness exerted a 
strongly positive influence on patients remaining in employment [47].

Furthermore, studies have shown that better disease control through the use of anti-TNFs ameliorates work disability in patients with PsA $[41,48,49]$, with the LOPAS II study demonstrating a 30\% improvement in presenteeism $(p<0.001)$ and $40 \%$ improvement in work productivity $(p<0.001)$ [48]. These findings suggest that work disability in active PsA is reversible with modern therapy, which could have major implications for both the individual as well as the wider society.

While it is recognized that WD is an important patient-outcome measure and several questionnaires measuring WD are available [50], no consensus exists on which tool to use. This is in part due to the poor correlation between these tools' ability to estimate components of WD [51] and lack of validation in PsA. The Work Productivity and Activity Impairment (WPAI) questionnaire is one such measure of work productivity. This questionnaire consists of six questions, asking patients to report the degree to which their condition, in this case PsA, has affected their ability to work and perform regular activities over the preceding week. Four outcome measures are generated (absenteeism, presenteeism, productivity loss, and general activity impairment). While the WPAI questionnaire is yet to be validated in PsA, its use is supported by the OMERACT (Outcomes Measures in Rheumatology) group [52] and it is the most patient-favored work-related measure, with $29 \%$ of patients reporting it as being the most relevant to them [52].

\section{Treatment Satisfaction in Psoriatic Arthritis}

The expansion of treatment options available to patients with PsA has led to significant improvements in patient outcomes. However, with this comes different modalities, frequencies of drug administration, and side-effects, which can considerably influence patient quality of life. One study found that $45.5 \%$ of patients with PsA are dissatisfied with their treatment [53]. Suboptimal medication adherence is known to impact the success of treatment outcomes, patient quality of life and has economic implications [54-56]. Patient satisfaction with treatment can predict adherence to medication regimens in chronic diseases, with convenience of use and side-effects being prominent predictors of adherence [57]. In addition, patient satisfaction with medications is positively correlated with health-related quality of life [57]. Assessment and understanding of patient preference, attitudes towards treatment, and medication satisfaction are necessary in ensuring uptake and adherence to these medications and improving patient outcomes.

The Treatment Satisfaction Questionnaire for Medication (TSQM) is a widely used, reliable, and validated instrument to assess patients' satisfaction with their treatment [58]. This questionnaire has been used in other rheumatic diseases and psoriasis [59-62]; however, to our knowledge has not been evaluated in PsA. The TSQM version 1.4 consists of 14 items, which explore four domains: side-effects, effectiveness, convenience, and global satisfaction with the medication. Each domain is scored 0-100, with higher scores indicating better treatment satisfaction. Abbreviated versions of TSQM have since been developed: TSQM Version II (11 items) [63] and TSQM-9 (9 items) [64]. Assessment of treatment satisfaction through the use of the TSQM provides an opportunity to understand and incorporate patients' perspectives into shared clinical decision-making and better identify those patients at risk of poor adherence. This enables clinicians to target their interventions towards the aspects responsible for poor concordance. PsA studies using validated measures in real-life clinical conditions are needed to assess patient satisfaction with conventional synthetic DMARDs and biological DMARDS. This will be critical in improving patient satisfaction and ultimately ensuring better adherence, patient outcomes, and health-related quality of life in psoriatic arthritis. 


\section{CONCLUSIONS}

It is well recognized that psoriatic disease is a complex condition, encompassing a multitude of clinical manifestations, which significantly impact patients' function and quality of life. In recent years, there has been a paradigm shift towards the development of disease activity indices that are responsive to clinical measures and also patient-reported outcomes, thereby capturing the full burden of disease. Widespread implementation of these instruments in routine clinical practice and research trials will be pivotal to providing clinicians with a better understanding of the full patient experience, in aiding treatment decisions and providing further details on patient satisfaction and efficacy of novel therapies in PsA.

\section{ACKNOWLEDGEMENTS}

Funding. No article processing charges were received by the journal for the publication of this article. Laura J. Tucker is funded by the Norman Collinson Foundation as a Clinical Research Fellow. Laura C. Coates is funded by a National Institute for Health Research Clinician Scientist award. The research was supported by the National Institute for Health Research (NIHR), Oxford and Leeds Biomedical Research Centres. The views expressed are those of the author(s) and not necessarily those of the NHS, the NIHR or the Department of Health.

Medical Writing and Editorial Assistance. No medical writing or editorial assistance was received during the writing of this article.

Authorship. All named authors meet the International Committee of Medical Journal Editors (ICMJE) criteria for authorship for this article, take responsibility for the integrity of the work as a whole, and have given their approval for this version to be published.
Disclosures. Laura J. Tucker, Laura C. Coates, and Philip S. Helliwell declare that they have nothing to disclose.

Compliance with Ethics Guidelines. This article is based on previously conducted studies and does not contain any studies with human participants or animals performed by any of the authors.

Data Availability. Data sharing is not applicable to this article, as no datasets were generated or analyzed during the current study.

Open Access. This article is distributed under the terms of the Creative Commons Attribution-NonCommercial 4.0 International License (http://creativecommons.org/licenses/ by-nc/4.0/), which permits any noncommercial use, distribution, and reproduction in any medium, provided you give appropriate credit to the original author(s) and the source, provide a link to the Creative Commons license, and indicate if changes were made.

\section{REFERENCES}

1. Orbai AM, et al. International patient and physician consensus on a psoriatic arthritis core outcome set for clinical trials. Ann Rheum Dis. 2016;76(4):673-80.

2. Coates LC, et al. Effect of tight control of inflammation in early psoriatic arthritis (TICOPA): a UK multicentre, open-label, randomised controlled trial. Lancet. 2015;386(10012):2489-98.

3. Coates LC, et al. Group for Research and Assessment of Psoriasis and Psoriatic Arthritis 2015 Treatment Recommendations for Psoriatic Arthritis. Arthritis Rheumatol. 2016;68(5):1060-71.

4. Gossec L, et al. European League Against Rheumatism (EULAR) recommendations for the management of psoriatic arthritis with pharmacological therapies: 2015 update. Ann Rheum Dis. 2016;75(3):499-510.

5. Smolen JS, et al. Treating axial spondyloarthritis and peripheral spondyloarthritis, especially psoriatic arthritis, to target: 2017 update of recommendations by an international task force. Ann Rheum Dis. 2018;77(1):3-17. 
6. Coates LC, et al. Reduced joint counts misclassify patients with oligoarticular psoriatic arthritis and miss significant numbers of patients with active disease. Arthritis Rheum. 2013;65(6):1504-9.

7. Fransen J, et al. Performance of response criteria for assessing peripheral arthritis in patients with psoriatic arthritis: analysis of data from randomised controlled trials of two tumour necrosis factor inhibitors. Ann Rheum Dis. 2006;65(10):1373-8.

8. Nell-Duxneuner VP, et al. Evaluation of the appropriateness of composite disease activity measures for assessment of psoriatic arthritis. Ann Rheum Dis. 2010;69(3):546-9.

9. Schoels MM, et al. Disease activity in psoriatic arthritis (PsA): defining remission and treatment success using the DAPSA score. Ann Rheum Dis. 2016;75(5):811-8.

10. Aletaha D, Alasti F, Smolen JS. Disease activity states of the DAPSA, a psoriatic arthritis specific instrument, are valid against functional status and structural progression. Ann Rheum Dis. 2017;76(2):418-21.

11. Healy PJ, Helliwell PS. Measuring clinical enthesitis in psoriatic arthritis: assessment of existing measures and development of an instrument specific to psoriatic arthritis. Arthritis Rheum. 2008;59(5):686-91.

12. Maksymowych WP, et al. Development and validation of the Spondyloarthritis Research Consortium of Canada (SPARCC) Enthesitis Index. Ann Rheum Dis. 2009;68(6):948-53.

13. D'Agostino MA, et al. Assessment of peripheral enthesitis in the spondylarthropathies by ultrasonography combined with power Doppler: a crosssectional study. Arthritis Rheum. 2003;48(2):523-33.

14. Healy PJ, Helliwell PS. Measuring dactylitis in clinical trials: which is the best instrument to use? J Rheumatol. 2007;34(6):1302-6.

15. Mease PJ, et al. Effect of certolizumab pegol on signs and symptoms in patients with psoriatic arthritis: 24-week results of a phase 3 double-blind randomised placebo-controlled study (RAPID-PsA). Ann Rheum Dis. 2014;73(1):48-55.

16. Eder L, et al. Is ASDAS better than BASDAI as a measure of disease activity in axial psoriatic arthritis? Ann Rheum Dis. 2010;69(12):2160-4.

17. Kilic G, et al. Comparison of ASDAS and BASDAI as a measure of disease activity in axial psoriatic arthritis. Clin Rheumatol. 2015;34(3):515-21.
18. Fredriksson T, Pettersson U. Severe psoriasis-oral therapy with a new retinoid. Dermatologica. 1978;157(4):238-44.

19. Walsh JA, et al. Product of the physician global assessment and body surface area: a simple static measure of psoriasis severity in a longitudinal cohort. J Am Acad Dermatol. 2013;69(6):931-7.

20. Merola JF, et al. Evaluation of sPGA x BSA as an outcome measure and treatment target for clinical practice. J Invest Dermatol. 2018;138(9):1955-61.

21. Coates LC, Fransen J, Helliwell PS. Defining minimal disease activity in psoriatic arthritis: a proposed objective target for treatment. Ann Rheum Dis. 2010;69(1):48-53.

22. Kavanaugh A, et al. Radiographic progression of patients with psoriatic arthritis who achieve minimal disease activity in response to golimumab therapy: results through 5 years of a randomized. Placebo-controlled study. Arthritis Care Res (Hoboken). 2016;68(2):267-74.

23. Queiro R, et al. Minimal disease activity and impact of disease in psoriatic arthritis: a Spanish cross-sectional multicenter study. Arthritis Res Ther. 2017;19(1):72.

24. Coates LC, et al. Minimal disease activity among active psoriatic arthritis patients treated with secukinumab: 2-year results from a multicenter randomized, double-blind, parallel-group. Arthritis Care Res (Hoboken): Placebo-Controlled Phase-III Study; 2018.

25. Coates LC, et al. Group for research and assessment of psoriasis and psoriatic arthritis/outcome measures in rheumatology consensus-based recommendations and research agenda for use of composite measures and treatment targets in psoriatic arthritis. Arthritis Rheumatol. 2018;70(3):345-55.

26. Mumtaz A, et al. Development of a preliminary composite disease activity index in psoriatic arthritis. Ann Rheum Dis. 2011;70(2):272-7.

27. Ritchlin CT, et al. Treatment recommendations for psoriatic arthritis. Ann Rheum Dis. 2009;68(9):1387-94.

28. Fitzgerald $\mathrm{O}$ et al. Application of composite disease activity scores in psoriatic arthritis to the PRESTA data set. Annal Rheumatic Diseases, 2011.

29. Helliwell PS et al. The development of candidate composite disease activity and responder indices for psoriatic arthritis (GRACE project). Annal Rheum Diseases 2012(online first 17/07/2012). 
30. Helliwell PS, Kavanaugh A. Comparison of composite measures of disease activity in psoriatic arthritis using data from an interventional study with golimumab. Arthritis Care Res (Hoboken). 2014;66(5):749-56.

31. Helliwell PS, Kavanaugh A. Radiographic progression in psoriatic arthritis achieving a good response to treatment: data using newer composite indices of disease activity. Arthritis Care Res (Hoboken). 2018;70(5):797-800.

32. Helliwell PS, FitzGerald O, Fransen J. Composite disease activity and responder indices for psoriatic arthritis: a report from the GRAPPA 2013 meeting on development of cutoffs for both disease activity states and response. J Rheumatol. 2014;41(6):1212-7.

33. Scoggins JF, Patrick DL. The use of patient-reported outcomes instruments in registered clinical trials: evidence from ClinicalTrials.gov. Contemp Clin Trials. 2009;30(4):289-92.

34. Kalyoncu U, et al. Reporting of patient-reported outcomes in recent trials in rheumatoid arthritis: a systematic literature review. Ann Rheum Dis. 2009;68(2):183-90.

35. Kirwan JR, et al. Updating the OMERACT filter: implications for patient-reported outcomes. J Rheumatol. 2014;41(5):1011-5.

36. Gossec L, et al. A patient-derived and patient-reported outcome measure for assessing psoriatic arthritis: elaboration and preliminary validation of the Psoriatic Arthritis Impact of Disease (PsAID) questionnaire, a 13-country EULAR initiative. Ann Rheum Dis. 2014;73(6):1012-9.

37. Holland R, et al. Validation of the Psoriatic Arthritis Impact of Disease (PsAID) Questionnaire and its potential as a single-item outcome measure in clinical practice. Ann Rheum Dis. 2018;77(3):343-7.

38. Di Carlo $\mathrm{M}$, et al. The 12-item Psoriatic Arthritis Impact of Disease Questionnaire: construct validity, reliability, and interpretability in a clinical setting. J Rheumatol. 2017;44(3):279-85.

39. Verstappen SM, et al. Working status in patients with rheumatoid arthritis, ankylosing spondylitis and psoriatic arthritis: results from the British Society for Rheumatology Biologics Register. Rheumatology (Oxford). 2010;49(8):1570-7.

40. Christophers E, et al. The risk of psoriatic arthritis remains constant following initial diagnosis of psoriasis among patients seen in European dermatology clinics. J Eur Acad Dermatol Venereol. 2010;24(5):548-54.
41. Kavanaugh A, et al. Effect of infliximab therapy on employment, time lost from work, and productivity in patients with psoriatic arthritis. J Rheumatol. 2006;33(11):2254-9.

42. Kaarela K, Lehtinen K, Luukkainen R. Work capacity of patients with inflammatory joint diseases. An eight-year follow-up study. Scand J Rheumatol. 1987;16(6):403-6.

43. Gottlieb $\mathrm{AB}$, et al. Clinical characteristics of psoriatic arthritis and psoriasis in dermatologists' offices. J Dermatolog Treat. 2006;17(5):279-87.

44. Radtke MA, et al. Prevalence and clinical features of psoriatic arthritis and joint complaints in 2009 patients with psoriasis: results of a German national survey. J Eur Acad Dermatol Venereol. 2009;23(6):683-91.

45. Zhu TY, et al. Socioeconomic burden of psoriatic arthritis in Hong Kong: direct and indirect costs and the influence of disease pattern. J Rheumatol. 2010;37(6):1214-20.

46. Tillett W, Vries C, McHugh NJ. Work disability in psoriatic arthritis: a systematic review. Rheumatology (Oxford). 2012;51(2):275-83.

47. Tillett W, et al. Factors influencing work disability in psoriatic arthritis: first results from a large UK multicentre study. Rheumatology (Oxford). 2015;54(1):157-62.

48. Tillett W, et al. Effect of anti-TNF and conventional synthetic disease-modifying anti-rheumatic drug treatment on work disability and clinical outcome in a multicentre observational cohort study of psoriatic arthritis. Rheumatology (Oxford). 2017;56(4):603-12.

49. Kristensen LE, et al. Long-term work disability in patients with psoriatic arthritis treated with antitumour necrosis factor: a population-based regional Swedish cohort study. Ann Rheum Dis. 2013;72(10):1675-9.

50. Beaton D, et al. Measuring worker productivity: frameworks and measures. J Rheumatol. 2009;36(9):2100-9.

51. Zhang W, et al. Productivity loss due to presenteeism among patients with arthritis: estimates from 4 instruments. J Rheumatol. 2010;37(9):1805-14.

52. Beaton DE, et al. OMERACT filter evidence supporting the measurement of at-work productivity loss as an outcome measure in rheumatology research. J Rheumatol. 2016;43(1):214-22. 
53. Armstrong AW, et al. Undertreatment, treatment trends, and treatment dissatisfaction among patients with psoriasis and psoriatic arthritis in the United States: findings from the National Psoriasis Foundation surveys, 2003-2011. JAMA Dermatol. 2013;149(10):1180-5.

54. Haynes $\mathrm{RB}$, et al. Interventions for enhancing medication adherence. Cochrane Database Syst Rev. 2008;2:p. Cd000011.

55. Hughes D, et al. Methods for integrating medication compliance and persistence in pharmacoeconomic evaluations. Value Health. 2007;10(6):498-509.

56. Taylor T. Understanding the choices that patients make. J Am Board Fam Med. 2000;13(2):124-33.

57. Delestras S, et al. Comparison between two generic questionnaires to assess satisfaction with medication in chronic diseases. PLoS One. 2013;8(2):e56247.

58. Atkinson MJ, et al. Validation of a general measure of treatment satisfaction, the Treatment Satisfaction Questionnaire for Medication (TSQM), using a national panel study of chronic disease. Health Qual Life Outcomes. 2004;2:12.

59. Jobanputra P et al. A randomised efficacy and discontinuation study of etanercept versus adalimumab (RED SEA) for rheumatoid arthritis: a pragmatic, unblinded, non-inferiority study of first TNF inhibitor use: outcomes over 2 years. BMJ Open, 2012 2(6):56.

60. Lin HY, et al. Etoricoxib improves pain, function and quality of life: results of a real-world effectiveness trial. Int J Rheum Dis. 2010;13(2):144-50.

61. Khanna PP, et al. Health-related quality of life and treatment satisfaction in patients with gout: results from a cross-sectional study in a managed care setting. Patient Prefer Adherence. 2015;9:971-81.

62. Schaarschmidt ML, et al. Treatment satisfaction of patients with psoriasis. Acta Derm Venereol. 2015;95(5):572-8.

63. Atkinson MJ, et al. Hierarchical construct validity of the treatment satisfaction questionnaire for medication (TSQM version II) among outpatient pharmacy consumers. Value Health. 2005;8(Suppl 1):S9-24.

64. Bharmal $\mathrm{M}$, et al. Validation of an abbreviated Treatment Satisfaction Questionnaire for Medication (TSQM-9) among patients on antihypertensive medications. Health Qual Life Outcomes. 2009; 7:36. 